\title{
Facilidades e Dificuldades à Autonomia Profissional de Enfermeiros no Cuidado de Pessoas com Feridas: Estudo de Representações Sociais
}

\author{
Facilities and Difficulties to the Professional Autonomy of Nurses Caring for People \\ with Wounds: a Study of Social Representations
}

\section{Facilidades y Dificultades de la Autonomía Profesional de los Enfermeros en Personas con Heridas: un Estudio de Representaciones Sociales}

\author{
Érick Igor dos Santos?', Jéssica Grativol Aguiar Dias de Oliveira', Raquel de Souza Ramos², \\ Aline Cerqueira Santos Santana da Silva ${ }^{1}$, Luísa dos Santos Belém¹, Aline Lima da Silva ${ }^{1}$
}

\begin{abstract}
RESUMO
A autonomia profissional pressupõe independências moral e intelectual para usufruir da capacidade de governar-se pelos próprios meios e tomar decisões livremente. Esta pesquisa teve por objetivo analisar as representações sociais elaboradas por enfermeiros sobre as dificuldades para estabelecer uma autonomia profissional no cuidado às pessoas com feridas. Trata-se de uma pesquisa qualitativa, descritiva e exploratória, delineada a partir da teoria de representações sociais em sua abordagem processual, realizada com 31 enfermeiros que desempenhavam suas funções em um hospital público municipal do estado do Rio de Janeiro. As entrevistas foram transcritas e submetidas à análise de conteúdo temática, instrumentalizada pelo software Nvivo 10. No pensamento social dos enfermeiros, se a ausência da comissão de curativos, a indisponibilidade de produtos variados para coberturas, o cerceamento da liberdade de atuação pela instituição e a ausência de protocolos prejudicam a plenitude da autonomia profissional, este quadro pode ser revertido pelo estabelecimento da comissão de curativos, fornecimento de coberturas para eles, provimento da liberdade de atuação hospitalar e desenvolvimento de protocolos institucionais. Os enfermeiros percebem fatores que podem dificultar o exercício da autonomia profissional, mas que podem ser solucionados, dependendo das modificações realizáveis no contexto institucional. Conclui-se haver dualidade da instituição na representação social dos sujeitos, que a abordam ora como facilitadora da autonomia profissional, ora enquanto sua inibidora, baseando-se no contexto laboral que promovem aos enfermeiros em suas atividades junto a pessoas com lesões de pele.
\end{abstract}

DESCRITORES: Autonomia profissional. Enfermagem. Psicologia social.

\footnotetext{
ABSTRACT

Professional autonomy assumes moral and intellectual independences to take advantage of the ability to govern themselves by their own means and to make decisions freely. This research aimed at analyzing social representations made by nurses on the facilities and difficulties in establishing professional autonomy in the care of people with wounds. This is a qualitative, descriptive and exploratory study, which was outlined based on the theory of social representations in its procedural approach, carried out with 31 nurses who performed their duties in a municipal public hospital in the state of Rio de Janeiro, Brazil. The interviews were transcribed and submitted to a thematic content analysis that was conducted through the Nvivo 10 software. In the social thinking of nurses, if the absence of a curative

${ }^{1}$ Universidade Federal Fluminense (UFF) - Rio de Janeiro (RJ), Brasil. Endereço para correspondência: Universidade Federal Fluminense, Polo Universitário de Rio das Ostras (UFF-PURO) - Rua Recife, s/n - Jardim Bela Vista - CEP: 28890-000 - Rio das Ostras (RJ), Brasil - E-mail: eigoruff@gmail.com 2Instituto Nacional do Câncer (INCA) - Rio de Janeiro (RJ), Brasil.

Artigo recebido em: 23/09/2015 - Aceito para publicação em: 28/10/2015
} 
commission, the unavailability of various dressing products, the restriction of freedom of action by the institution, and the absence of protocols hinder the professional autonomy fullness, this situation can be reversed by the establishment of a dressing commission, providing bandages for dressings, provision of freedom for hospital operations, and development of institutional protocols. Nurses perceive factors that may difficult the exercise of professional autonomy, but which could be solved, depending on achievable changes in the institutional context. In conclusion, there is institutional duality in the subjects' social representations, which sometimes addresses it as a facilitator of professional autonomy while inhibiting it, depending on the work context that promotes to nurses in their activities with patients showing a skin lesion.

DESCRIPTORS: Professional autonomy. Nursing. Psychology, Social.

\section{RESUMEN}

La autonomía profesional presupone las independencias moral e intelectual para beneficiarse de la capacidad de gobernarse a sí mismos, por sus propios medios, y tomar decisiones libremente. Esta investigación tuvo como objetivo analizar las representaciones sociales realizadas por enfermeros sobre las dificultades en el establecimiento de una autonomía profesional en el cuidado de personas con heridas. Es una investigación cualitativa, descriptiva y exploratoria, usando la teoría de las representaciones sociales en su enfoque de procedimiento, realizada con 31 enfermeros que trabajaban en un hospital público municipal en el estado de Río de Janeiro, Brasil. Las entrevistas fueron transcritas y sometidas al análisis de contenido temático, instrumentalizado mediante el software NVivo 10. En el pensamiento social de los enfermeros, si la ausencia de la comisión de los curativos, la falta de diversos productos para coberturas, la restricción de la libertad de acción de la institución y la ausencia de protocolos impiden la plenitud de autonomía profesional, esta situación puede revertirse mediante el suministro de curativos, las coberturas para curativos, la libertad de desempeño de hospitales y el desarrollo de protocolos institucionales. Los enfermeros perciben factores que pueden obstaculizar el ejercicio de la autonomía profesional, pero que pueden resolverse, dependiendo de los cambios realizables en el contexto institucional. En conclusión, existe una dualidad de la institución en la representación social de los sujetos, que la aborda a veces como facilitadora de la autonomía profesional, o como la suya inhibidora, según el contexto de trabajo que promuevan los enfermeros en sus actividades con personas con lesiones en la piel.

DESCRIPTORES: Autonomía profesional. Enfermería. Psicología social.

\section{INTRODUÇÃO}

As feridas podem ser denominadas alterações da integridade anatômica da pele, resultantes de eventos traumáticos, vasculares, infecciosos ou neoplásicos. O tratamento delas envolve aspectos sistêmicos e locais, que devem ser, preferencialmente, desenvolvidos pela equipe multiprofissional.

Apesar disto, o tratamento e a prevenção de feridas, frequentemente, estão sob a responsabilidade do enfermeiro, sendo de sua competência a avaliação e a prescrição das melhores coberturas para o tratamento da lesão. A este profissional cabe executar, orientar ou supervisionar a equipe de Enfermagem na execução do curativo. $\mathrm{O}$ cuidado com feridas requer conhecimento específico, habilidade e autonomia.

Entende-se por expressão da autonomia profissional do enfermeiro quando este, dotado de independências moral e intelectual, usufrui da capacidade de governar-se pelos próprios meios e toma decisões livremente, estabelecendo sua prática individual ou coletiva. A autonomia profissional pressupõe competência e liberdade para se proceder às escolhas conscientes, dentre as opções possíveis ${ }^{1,2}$.

A autonomia profissional do enfermeiro no tratamento de feridas tem sido, ao longo do tempo e da evolução da Enfermagem, um tema importante à compreensão, tanto pela premência da definição de soluções aos seus desafios quanto pela necessidade de investigar a forma com a qual os enfermeiros se relacionam entre si, com a equipe de saúde e com a sociedade em geral, o que fornece a aproximação necessária para a formação de representações sociais da Enfermagem, do enfermeiro, de seu papel social e da sua autonomia profissional pelos mais diversos grupos sociais ${ }^{1-3}$.

As representações sociais são uma forma de conhecimento, que é socialmente elaborada e compartilhada, tendo visão prática e concorrendo para a construção de uma realidade comum a um conjunto social. Também pode-se afirmar que representar é atribuir conhecimentos de caráter coletivo a alguma coisa ou alguém. A representação é constituída por um conjunto de crenças, informações, opiniões e atitudes a propósito de um dado objeto social. A teoria 
das representações sociais é abrangente e permite o entendimento das dimensões da realidade, além de buscar estudar diferentes espécies de atitudes humanas ${ }^{4}$.

A relevância deste estudo reside na assertiva de que esta teoria pode ser utilizada a fim de entender o processo de formação dos conhecimentos sobre uma dada profissão; seus aspectos técnicos e limites de atuação; como esta consegue estruturar-se histórica e socioculturalmente, bem como é ressignificada por um determinado grupo social, considerando as dificuldades, os dilemas e os desafios atuais enfrentados pela profissão para sua afirmação.

\section{OBJETIVO}

Este estudo teve por objetivo analisar as representações sociais elaboradas por enfermeiros sobre as facilidades e as dificuldades para estabelecer a autonomia profissional no cuidado às pessoas com feridas.

\section{MÉTODOS}

Trata-se de uma pesquisa descritiva e exploratória, de abordagem qualitativa, realizada com 31 enfermeiros que trabalhavam em um hospital municipal da região dos Lagos do estado do Rio de Janeiro, entre janeiro e março de 2015. É importante destacar que o cenário em questão, por diversos fatores políticos e econômicos, apresentava dificuldades na manutenção da comissão de curativos efetivamente funcional e operante.

Adotou-se o referencial teórico-metodológico das representações sociais em sua abordagem processual, a qual preocupa-se, centralmente, com a construção da representação, seus processos de elaboração e os aspectos que a constituem, tais como crenças, valores, opiniões, elementos culturais e ideológicos ${ }^{5}$.

Os critérios de inclusão foram enfermeiros que desenvolviam suas atividades laborais no cenário do estudo por, no mínimo, seis meses e que aceitaram gratuitamente e conscientemente participar desta pesquisa. Os critérios de exclusão estabelecidos foram enfermeiros em afastamento, férias, licença-maternidade ou que não tiveram disponibilidade de tempo para participar da pesquisa, mesmo após sucessivas tentativas do agendamento de entrevista.

Mediante a amostragem por conveniência, houve a tentativa de alcance de $100 \%$ dos enfermeiros, no universo amostral de 50 profissionais, para averiguação de quais se encaixavam nos critérios de inclusão. Deles, apenas 31 profissionais se encaixaram nos critérios supracitados, sendo este um número aceitável para trabalhos em representações sociais na perspectiva da abordagem processual ${ }^{6}$.

Buscou-se atender à Resolução 466/12 do Conselho Nacional de Saúde. O projeto ao qual esta pesquisa está vinculada obteve aprovação sob o parecer 924.334. A participação dos sujeitos foi facultativa, sendo convidados por meio do termo de consentimento livre e esclarecido, que demandou a sua assinatura.

As técnicas escolhidas para a coleta de dados foram o questionário, contendo dados sociodemográficos para conhecer o perfil dos enfermeiros, além da entrevista semiestruturada em profundidade.

Após a coleta de dados, as entrevistas foram transcritas e analisadas por meio da técnica de análise de conteúdo temática, que propõe categorização sistemática do discurso dos entrevistados, possibilitando a classificação e a quantificação ${ }^{7}$. O software utilizado para a análise de dados das entrevistas foi o QSR Nvivo 10, que se propõe a reunir, organizar e sistematizar conteúdos de entrevistas, discussões em grupo, artigos científicos, áudios e vídeos, sem requerer preparo prévio do corpus de análise ${ }^{8}$. A análise do questionário sociodemográfico foi realizada por meio de porcentagem.

\section{RESULTADOS}

Os sujeitos eram, em sua maioria, do sexo feminino (90\%); pertencentes à faixa etária de 25 a 34 anos (39\%) e 35 a 44 anos (39\%); de religião católica (52\%); com companheiro (68\%); pós-graduados lato sensu (94\%); com $\leq 5$ anos de atuação institucional (65\%); com $\leq 5$ anos de atuação profissional como enfermeiro (38\%); com renda pessoal $\leq 6$ salários-mínimos (42\%); apenas um emprego (64\%); carga horária semanal de 24 horas (84\%); com nenhuma formação fora da área de Enfermagem (81\%); função de enfermeiro assistencial (68\%) e acesso a informações científicas (100\%). Dos 31 profissionais, 58\% relataram ter autonomia profissional.

$\mathrm{O}$ resultado da análise instrumentalizada pelo $N V i v o$ 10 obteve 573 unidades de registro, distribuídas em quatro categorias, as quais representam 100\% do corpus investigado. Neste estudo, será aprofundada a categoria número dois, 
que apresenta 151 unidades de registro e 72 temas, denominada "Facilidades e dificuldades para estabelecer autonomia profissional".

Os recortes de discurso dos sujeitos que melhor ilustram esta categoria serão apresentados, seguidos do número atribuído a cada enfermeiro participante da pesquisa e do seu gênero declarado no questionário de caracterização sociodemográfica.

A valorização da comissão de curativos é uma das temáticas destacadas dentro desta classe, na qual os enfermeiros expressaram a importância de suas atividades voltadas para educação permanente e constante atualização no cuidado à pessoas com feridas, sendo, desta maneira, um fator facilitador à autonomia profissional. Isto ficou caracterizado nos recortes discursivos a seguir.

Enfermeira 1 - Para mim, é excelente ter pessoas mais especializadas para avaliar, sempre atualizadas, porque às vezes nós não conseguimos ter essa atualização sempre. É sempre bom ter essa comissão para nos manter atualizados.

Enfermeira 4 - Pois, com a comissão de curativos, temos mais capacitação. Eles podem sempre se atualizar, passar para os enfermeiros que estão atuando. Isso pode até encurtar o tempo de fechamento de uma ferida.

Enfermeira 26 - A comissão de curativos oferece suporte para que nós estejamos bem informados, bem atualizados e para que tenhamos maior qualidade de assistência.

Os participantes, portanto, posicionam-se favoravelmente à presença da comissão de curativos, o que evidencia uma dimensão atitudinal positiva da representação. Há valorização da comissão de curativos como um fator facilitador à autonomia e, nesta perspectiva, os enfermeiros atribuem a sua autonomia profissional, no cuidado às pessoas acometidas por feridas, ao nível de conhecimento e capacitação proporcionados tal atividade.

Os informantes evidenciaram as influências que a instituição pode exercer sobre o seu trabalho e o de sua equipe.

Enfermeira 4-As dificuldades são as instituiçôes. Muitas das vezes, nós vamos contra o que a administração quer, porque temos autonomia. O Coren [Conselho Regional de Enfermagem] nos dá autonomia para exercer nossas funções, mas às vezes a administração do hospital diz que isso não é serviço da Enfermagem.
Enfermeira 18 - Temos que ter uma instituição que nos permita ter autonomia, porque muita gente detém o conhecimento, mas não tem autonomia nenhuma, porque a própria instituição não permite isso.

Enfermeiro 30 - [...] também depende do que o hospital tem a oferecer, porque não adianta conhecermos o suprassumo das coberturas e o hospital só te oferece AGE [ácidos graxos essenciais] e desbridantes enzimáticos. Então não adianta nada, só nos resta utilizá-las de modo adequado e satisfatório.

Percebe-se uma dualidade na organização do pensamento social dos sujeitos. A instituição, quando é permeável ao livre exercício profissional e fornece recursos materiais aos enfermeiros, no cuidado às pessoas com feridas, favorece a sua autonomia profissional; caso contrário, a desfavorece. A partir do que foi exposto pelos enfermeiros, percebe-se que os participantes questionam sua própria autonomia profissional quando a instituição restringe sua atuação, contradizendo a estrutura ético-legal, oriunda do conselho da profissão sobre uma determinada conduta no tocante ao cuidado no tratamento e à prevenção de feridas. Desse modo, a instituição pode interferir, de acordo com os sujeitos, positiva ou negativamente, na qualidade da assistência prestada.

Os enfermeiros acreditam ser um fator dificultador à sua autonomia profissional a falta de um protocolo que guie, dentro da instituição, as práticas do enfermeiro quanto ao cuidado às pessoas com feridas, como pode ser averiguado nos seguintes recortes de discurso a seguir:

Enfermeira 20 - Quando vira rotina, ninguém vai modificar aquilo que nós já escrevemos e o hospital já estabeleceu como protocolo.

Enfermeiro 27 - Falta um protocolo, falta um procedimento operacional padrão. Isso dificulta o nosso trabalho porque a partir do momento em que temos um protocolo, todos seguem e respeitam. Então, às vezes, é preciso que a instituição padronize os curativos e então todos nós teríamos mais autonomia quanto a isso.

Entre os conteúdos representacionais dos participantes, a falta de um documento que sirva de norteador à indicação de coberturas é um fator que dificulta o estabelecimento da autonomia profissional, pois as suas ações sem este recurso ficam sujeitas à interferência de outros profissionais de saúde. 


\section{DISCUSSÃO}

Para os entrevistados, um fator facilitador à sua autonomia profissional, frente ao cuidado de pessoas com feridas, é a implementação de uma comissão de curativos. Esta é importante, pois é formada por profissionais mais capacitados e especializados que promovem a atualização dos enfermeiros que atuam nas clínicas. Desse modo, a comissão de curativos contribui, indiretamente, para uma maior especialização do cuidado, fornecendo apoio técnico e conhecimento científico, o que atribui qualidade à assistência. É necessário conhecer para cuidar melhor e cuidar para confrontar. Assim, conhecimento, poder e autonomia se tencionam para libertar o fazer humano das amarras que o impedem de concretizar ações possíveis, sendo o saber essencial ao desenvolvimento de uma atenção de qualidade na assistência de Enfermagem?

A elaboração de uma comissão de curativo como plataforma de trabalho tem por objetivo a prevenção, o tratamento e o controle das taxas das lesões de pele no ambiente hospitalar. Tais ferimentos tornam-se um transtorno para os pacientes internados e seus familiares e demandam horas mais longas de cuidado para a equipe de Enfermagem, retardando, em alguns casos, o tempo de hospitalização, o que eleva os custos hospitalares ${ }^{10}$. A comissão de curativos torna-se responsável pela elaboração de regimentos, protocolos, instrumentos para acompanhamento das feridas, capacitação profissional dos enfermeiros para a aplicação destes instrumentos e monitoramento das medidas adotadas.

Um aspecto que afeta negativamente o estabelecimento da autonomia profissional do enfermeiro é a interferência direta de outros profissionais, principalmente médicos, na prescrição dos produtos de uso tópico no manejo das lesões de pele. Sendo assim, essa interferência não construtiva pode ser contornada por meio da implantação de protocolos. A relação entre enfermeiros e médicos possui um contexto histórico na formação de atribuições e competências de cada profissão ${ }^{11}$. Uma pesquisa com estudantes de Enfermagem demonstrou a visão de centralização no saber médico como um obstáculo à formação e ao trabalho profissional de Enfermagem ${ }^{12}$.

Em um estudo que buscou a legislação brasileira específica sobre a autonomia do enfermeiro no cuidado às feridas, notou-se um déficit de normas por parte dos conselhos regionais de Enfermagem ${ }^{6}$. Esse dado sustenta a necessidade do uso de protocolos institucionais pelos enfermeiros no cuidado de pessoas com feridas. Para este profissional prestar uma assistência mais autônoma, é preciso objetivar, aperfeiçoar e padronizar os procedimentos de prevenção e tratamento de feridas. Isso deve ser realizado perante protocolos técnicos que garantam respaldos legal, técnico e científico ao profissional, a fim de aprimorar a assistência ao portador de feridas ${ }^{6}$.

Outro fator interveniente para a autonomia do enfermeiro, de acordo com os participantes, é a própria dinâmica institucional na qual eles trabalham, que pode ser cerceadora. Para eles, frequentemente, o hospital restringe atribuições que competem ao enfermeiro diante de um determinado procedimento, apesar de sua autonomia ser garantida pela Lei 7.498/86. Outra consideração a respeito da instituição, porém relacionada diretamente ao cuidado de feridas, é sobre a disponibilidade de materiais e coberturas necessários para o cuidado. A restrição de coberturas é apontada como um fator dificultador para o exercício pleno da autonomia. Tais dados são corroborados por outra pesquisa conduzida, que demonstrou, por meio dos depoimentos de membros da equipe de Enfermagem, fatores que interferem no desempenho profissional, como a falta de recursos materiais ${ }^{13}$.

Neste estudo, as representações elaboradas por enfermeiros reúnem conteúdos que favorecem ou desfavorecem o estabelecimento de autonomia profissional, numa relação de balanço e contrabalanço. Portanto, os enfermeiros percebem fatores que podem dificultar o exercício da autonomia, mas que podem ser solucionados, a depender de modificações realizáveis no contexto institucional. Logo, no pensamento social dos enfermeiros, se a ausência da comissão de curativos, a indisponibilidade de produtos variados para coberturas, o cerceamento da liberdade de atuação pela instituição e a ausência de protocolos prejudicam a plenitude da autonomia profissional, este quadro pode ser revertido pelo estabelecimento da comissão de curativos, fornecimento de coberturas para o curativo, provimento de liberdade de atuação hospitalar e desenvolvimento de protocolos institucionais (Figura 1).

Esta pesquisa possui fragilidades, como ter sido desenvolvida em um único cenário, com um número restrito de sujeitos e em um período específico. Contudo, sua principal potencialidade é promover reflexões quanto às políticas institucionais voltadas ao trabalho do enfermeiro, as quais podem subsidiar propostas de mudanças no processo de trabalho dos enfermeiros no cuidado às pessoas com feridas ${ }^{14}$. 


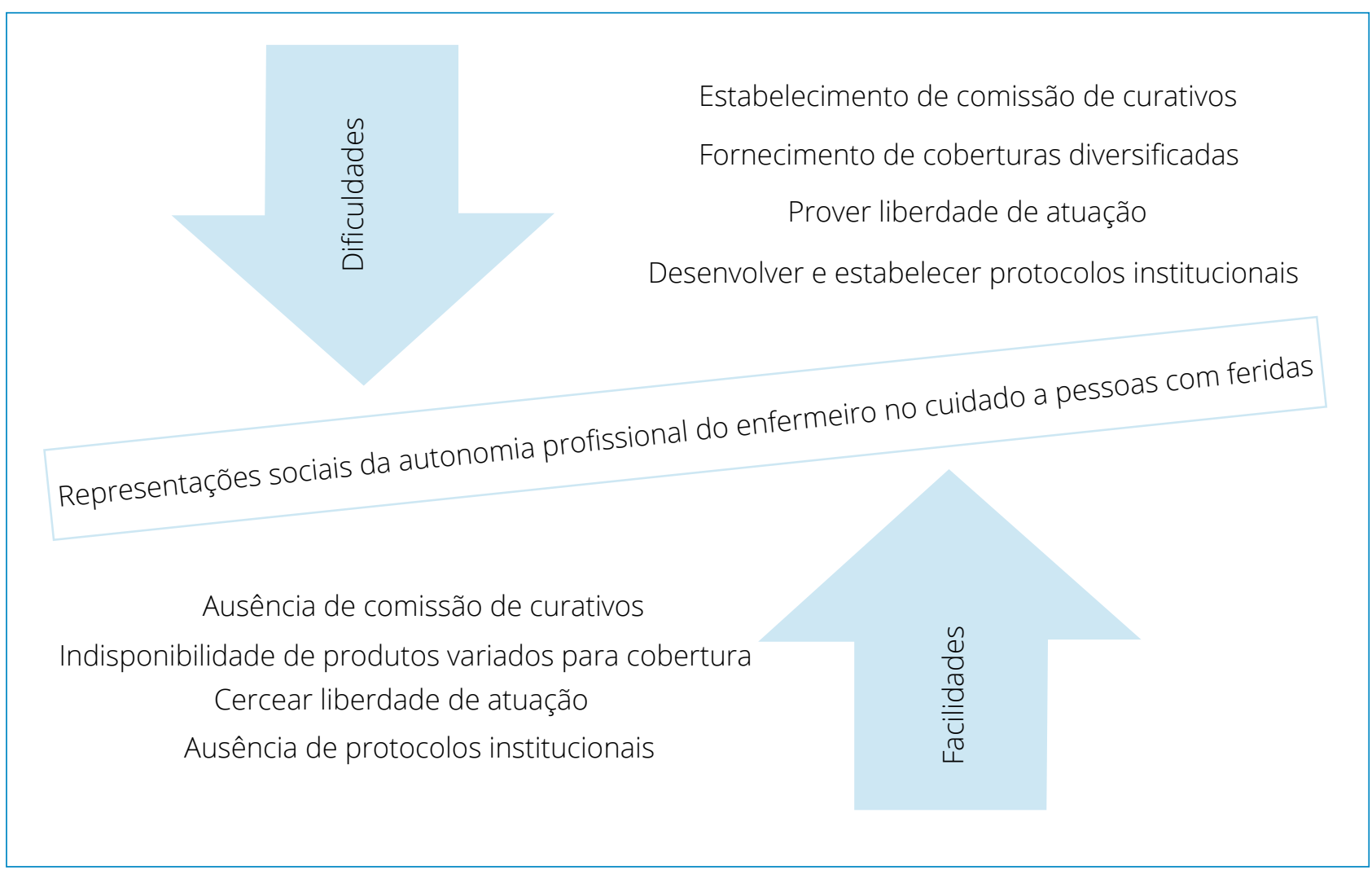

Figura 1. Ilustração da dualidade do papel institucional nas representações sociais da autonomia profissional elaboradas por enfermeiros. Rio das Ostras, Rio de Janeiro, Brasil, 2015.

\section{CONCLUSÃO}

Conclui-se que as representações elencadas nesta pesquisa podem nortear ações das instituições de saúde que viabilizem o fortalecimento de autonomia profissional do enfermeiro, sob a égide ético-legal da Enfermagem, na esfera dos conselhos regionais e federal, bem como na legislação vigente.

Ressalta-se que as medidas, as quais, para os sujeitos, potencializam sua autonomia profissional, caso aplicadas, além de serem de baixo custo, contribuem não somente para um maior grau de satisfação profissional, mas para a retenção do mesmo na instituição, redução dos custos hospitalares, maior grau de segurança do paciente e potencialização da qualidade da assistência prestada.

Os dados obtidos apontam para uma dualidade da instituição no pensamento social dos sujeitos, ora como facilitadora da autonomia profissional, quando fornece os requisitos básicos para o seu estabelecimento, ora enquanto sua inibidora, ao fornecer um contexto insatisfatório para os enfermeiros em suas atividades laborais junto a pessoas com lesões de pele.

Este estudo teve seu objetivo alcançado, e ficaram evidenciadas a usabilidade, a adequação e a pertinência da teoria das representações sociais aos estudos desta natureza.

Quanto às lacunas do conhecimento, ficou evidenciada a necessidade da realização de pesquisas que abordem o objeto 'autonomia profissional do enfermeiro' na prevenção e no tratamento de feridas, sob múltiplos e diversificados olhares teóricos e metodológicos. No caso dos estudos de representações sociais, é possível, por exemplo, replicar esta pesquisa em outras instituições de saúde para reiterar ou refutar os dados obtidos, sendo possível ampliá-la aos dirigentes das unidades de saúde para identificar possíveis obstáculos psicossociais, econômicos ou culturais que se coloquem como empecilhos à autonomia profissional do enfermeiro. 


\section{REFERÊNCIAS}

1. Bueno FM, Queiroz MS. O enfermeiro e a construção da autonomia profissional no processo de cuidar. Rev Bras Enfermagem. 2006;59(2):222-7.

2. Berti HW, Braga EM, Godoy IW, Spiri C, Bocchi SC. Percepção de enfermeiros recém-graduados sobre sua autonomia profissional e sobre o processo de tomada de decisão do paciente. Rev Latino-am Enfermagem. 2008;16(2):184-91.

3. Ferreira AM, Bogamil DD, Tormenta PC. O enfermeiro e o tratamento de feridas: em busca da autonomia do cuidado. Arq Cienc Saúde. 2008;15(3):105-9.

4. GomesAMT, Oliveira DC. O Núcleo central das representações de enfermeiros acerca da enfermagem: o papel próprio da profissão. Rev Enferm UERJ. 2010;18(3):352-8.

5. Arruda A. Teoria das representações sociais e teorias de gênero. Cad Pesq. 2002;117(127):127-47.

6. Gil AC. Métodos e técnicas de pesquisa social. 6. ed. São Paulo: Atlas; 2009.

7. Oliveira DC. Análise de conteúdo temático-categorial: uma proposta de sistematização. Rev Enferm UERJ. 2008;16(4):569-76.

8. Taddeo OS, Gomes KW, Caprara A, Gomes AM, Oliveira GC, Moreira TM. Acesso, prática educativa e empoderamento de pacientes com doenças crônicas. Cienc Saúde Coletiva. 2012;17(11):2923-30.

9. Pires MR. Politicidade do cuidado e processos de trabalho em saúde: conhecer para cuidar melhor, cuidar para confrontar e cuidar para emancipar. Rev Latino-am Enfermagem. 2005;13(5):729-36.

10. Madeira FM, De Souza CJ. Elaboração do protocolo em assistência de enfermagem ao paciente portador de lesões de pele. Rev Enf Profissional. 2014;1(2):511-20.

11. Pires D. A enfermagem enquanto disciplina, profissão e trabalho. Rev Bras Enfermagem. 2009;62(5):739-44.

12. Ribeiro AA, Borenstein MS. A percepção dos formandos a respeito dos instrumentos básicos de enfermagem e sua aplicabilidade. Rev Bras Enfermagem. 2005;58(6):653-8.

13. Beserra FM, Souza AM, Moreira DA, Alves MD, Alencar BP. Significado do trabalho dos profissionais de enfermagem no hospital. Rev Enfermagem. 2010;28(2):31-9.

14. Santos Él. Cuidado e prevenção das skin tears por enfermeiros: revisão integrativa de literatura. Rev Gaúcha Enfermagem. 2014;35(2):142-9. 